\title{
NOTE \\ Virology \\ Rapid diagnosis of equine influenza by highly sensitive silver amplification immunochromatography system
}

\author{
Takashi YAMANAKA ${ }^{1) *}$, Manabu NEMOTO'1), Hiroshi BANNAI ${ }^{1)}$, \\ Koji TSUJIMURA ${ }^{1)}$, Takashi KONDO'), Tomio MATSUMURA'), Tao Qi Huang FU'), \\ Charlene Judith FERNANDEZ ${ }^{2)}$, Sarah GILDEA ${ }^{3)}$ and Ann CULLINANE')

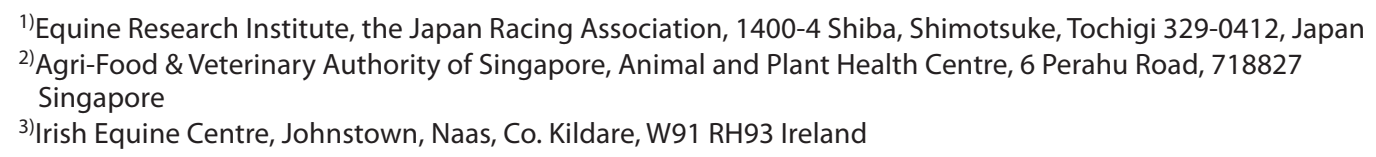

J. Vet. Med. Sci.

79(6): 1061-1063, 2017

doi: 10.1292/jvms.17-0105

Received: 1 March 2017

Accepted: 19 April 2017

Published online in J-STAGE:

1 May 2017
ABSTRACT. Equine influenza (EI) is a respiratory disease caused by equine influenza A virus (EIV, H3N8) infection. Rapid diagnosis is essential to limit the disease spread. We previously reported that some rapid antigen detection (RAD) tests are fit for diagnosing El although their sensitivity is not optimal. Here, we evaluated the performance of the newly developed RAD test using silver amplification immunochromatography (Quick Chaser Auto Flu A, B: QCA) to diagnose El. The detection limits of QCA for EIVs were five-fold lower than the conventional RAD tests. The duration of virus antigen detection in the infected horses was longer than the conventional RAD tests. We conclude that QCA could be a valuable diagnostic method for El.

KEY WORDS: diagnosis, equine influenza, rapid antigen detection, silver amplification

Equine influenza (EI) is caused by equine influenza A virus (EIV) infection and is one of the most important respiratory diseases of horses due to its high contagiousness [1]. EIV is a member of the family Orthomyxoviridae of the genus Influenzavirus A [4]. Although two subtypes of EIV have been recognized (H7N7 and H3N8), viruses of the H7N7 subtype have not been isolated for the last three decades and may be extinct [1]. In contrast, viruses of the H3N8 subtype are a major cause of respiratory disease in horses throughout the world [1]. Florida sublineage viruses of the H3N8 subtype have been responsible for all confirmed outbreaks of EI in recent years and since 2010 are the only viruses recommended for inclusion in EI vaccines by the OIE. This sublineage has diverged into antigenically two different clades, Florida sublineage Clade (Fc) 1 and Fc2. The former viruses predominate on the American continent but have caused outbreaks in Africa, Asia and Australia, and the latter viruses predominate on the Eurasian continent [1]. Horses infected with EIV show the acute onset of pyrexia, associated with depression, anorexia, nasal discharge and coughing. EIV infection occurs via the inhalation of aerosolized virus particle in the same way as human seasonal influenza [5].

A presumptive diagnosis of EI can be made in a group of susceptible horses on the basis of rapid spread of a febrile disease with frequent coughing [5]. However, as those clinical signs alone are not specific enough, laboratory tests to identify EIV in the respiratory secretions are needed to make a definitive diagnosis. We previously reported that some rapid antigen detection (RAD) tests are substantially fit for diagnosing EI but with the sensitivities ranging from 67 to $73 \%$ [8], so there still remains room for improvement. As the RAD tests quickly and easily provide results within $15 \mathrm{~min}$ in clinical practice, they allow veterinary clinicians to implement prompt sanitary measures to limit the disease spread, for example movement ban on horses. Also, the RAD tests have been utilized for large scale surveillance to give an overview of disease spread during an EI outbreak [6]. Recently, the RAD test using silver amplification immunochromatography, Quick Chaser Auto Flu A, B (QCA, Mizuho Medy, Saga, Japan) was licensed to diagnose human seasonal influenza in Japan [2, 3]. It was reported that the detection limits of QCA for influenza A viruses (H1N1, H1N1pdm and H3N2) decrease eight-fold and the diagnostic sensitivity is higher than conventional RAD tests [2]. In turn, the diagnostic accuracy of EI is also expected to be improved by QCA compared to the conventional RAD tests. Here, we assessed the diagnostic performance of QCA for EI compared with the conventional RAD tests.

Five-fold serial dilutions of A/equine/Malaysia/M201-1/2015 (H3N8) (M15, 10 ${ }^{7.0}$ egg infectious dose (EID) ${ }_{50} / \mathrm{ml}$, Fc1, http:// www.oie.int/our-scientific-expertise/specific-information-and-recommendations/equine-influenza/) and A/equine/Kildare/2/2010

*Correspondence to: Yamanaka, T., Equine Research Institute, the Japan Racing Association, 1400-4 Shiba, Shimotsuke, Tochigi 329-0412, Japan. e-mail: yamanaka@equinst.go.jp

@2017 The Japanese Society of Veterinary Science

This is an open-access article distributed under the terms of the Creative Commons Attribution Non-Commercial No Derivatives (by-ncnd) License. (CC-BY-NC-ND 4.0: https://creativecommons.org/licenses/by-nc-nd/4.0/) 
Table 1. Comparison of detection limits ${ }^{\mathrm{a})}$ to A/equine/ Malaysia/M201-1/2015 (H3N8) a representative of the Florida sublineage Clade 1 and A/equine/ Kildare/2/2010 (H3N8) a representative of the Florida sublineage Clade 2, of Quick Chaser Auto Flu A, B (QCA) and the two conventional RAD tests

\begin{tabular}{cll}
\hline Products & $\mathrm{M} 15^{\mathrm{e})}$ & $\mathrm{K} 10^{\mathrm{f}}$ \\
\hline $\mathrm{QCA}$ & $3.5^{\mathrm{a})}$ & 4.2 \\
$\mathrm{QC}^{\mathrm{b})}$ & 4.2 & $4.9^{\mathrm{d})}$ \\
$\mathrm{EMN}^{\mathrm{c})}$ & 4.2 & $4.9^{\mathrm{d})}$ \\
\hline
\end{tabular}

a) Detection limit $\left(\log (10) \mathrm{EID}_{50} / \mathrm{m} l\right)$ for each RAD test was determined using $100 \mu l$ of a five-fold serial dilution of the virus stock. b) Quick Chaser Flu A, B. c) BD Flu Examan. d) The data were cited from our previous study [8]. e) A/equine/Malaysia/M201-1/2015 (H3N8). f) A/equine/ Kildare/2/2010 (H3N8).
Table 2. Comparison of the test results of Quick Chaser Auto Flu A, B (QCA) with those of virus isolation (VI), the three molecular tests and the two conventional RAD tests [Quick Chaser (QC) and BD Flu Examan (EMN)] in the detection of EIV in the four horses experimentally infected with A/equine/ Malaysia/M201-1/2015 (H3N8), Florida sublineage Clade 1

\begin{tabular}{ccccccccc}
\hline $\begin{array}{c}\text { Days after } \\
\text { inoculation }\end{array}$ & $\begin{array}{c}\text { Clinical } \\
\text { signs }\end{array}$ & RT-PCR & rRT-PCR & RT-LAMP & QCA & VI & QC & EMN \\
\hline 0 & 0 & 0 & 0 & 0 & 0 & 0 & 0 & 0 \\
1 & 0 & 4 & 4 & 4 & 4 & 4 & 2 & 1 \\
2 & 4 & 4 & 4 & 4 & 4 & 4 & 4 & 4 \\
3 & 0 & 4 & 4 & 4 & 4 & 4 & 4 & 4 \\
4 & 2 & 4 & 4 & 4 & 4 & 4 & 4 & 4 \\
5 & 4 & 4 & 4 & 4 & 4 & 4 & 4 & 4 \\
6 & 4 & 4 & 4 & 4 & 4 & 3 & 4 & 4 \\
7 & 4 & 4 & 4 & 4 & 3 & 0 & 2 & 1 \\
8 & 2 & 2 & 3 & 3 & 1 & 0 & 0 & 0 \\
9 & 4 & 3 & 3 & 3 & 0 & 0 & 0 & 0 \\
10 & 0 & 3 & 3 & 3 & 0 & 0 & 0 & 0 \\
\hline
\end{tabular}

The days of sample collection are listed in the first column. The second column lists the number of horses that showed the clinical signs $\left(\geq 38.5^{\circ} \mathrm{C}\right.$ of rectal temperature and/or nasal discharge and/or coughing). The remaining columns list the number of horses that tested positive by each test.

Table 3. Comparison of the test results of QCA with VI, the three molecular tests and the two conventional RAD tests in the detection of EIV in the three horses experimentally infected with A/equine/Kildare/2/2010 (H3N8), Florida sublineage Clade 2

\begin{tabular}{ccccccccc}
\hline $\begin{array}{c}\text { Days after } \\
\text { inoculation }\end{array}$ & $\begin{array}{c}\text { Clinical } \\
\text { signs }^{\text {a }}\end{array}$ & RT-PCR $^{\text {a }}$ & rRT-PCR $^{\text {a) }}$ & RT-LAMPa) & QCA & VI $^{\text {a) }}$ & QC $^{\text {a) }}$ & EMN $^{\text {a) }}$ \\
\hline 0 & 0 & 0 & 0 & 0 & 0 & 0 & 0 & 0 \\
1 & 0 & 3 & 3 & 3 & 3 & 3 & 1 & 0 \\
2 & 3 & 3 & 3 & 3 & 3 & 3 & 3 & 3 \\
3 & 3 & 3 & 3 & 3 & 3 & 2 & 3 & 3 \\
4 & 3 & 3 & 3 & 3 & 3 & 3 & 3 & 3 \\
5 & 3 & 3 & 3 & 3 & 3 & 3 & 3 & 2 \\
6 & 3 & 3 & 3 & 3 & 3 & 2 & 2 & 2 \\
7 & 3 & 1 & 2 & 2 & 1 & 0 & 0 & 0 \\
8 & 3 & 0 & 1 & 0 & 0 & 0 & 0 & 0 \\
9 & 3 & 0 & 0 & 0 & 0 & 0 & 0 & 0 \\
10 & 2 & 0 & 0 & 0 & 0 & 0 & 0 & 0 \\
\hline
\end{tabular}

The days of sample collection are listed in the first column. The second column lists the number of horses that showed the clinical signs. The definition of the clinical signs and abbreviations in the first row are the same as in Table 2. The remaining columns list the number of horses that tested positive by each test. a) These data were cited from our previous study [8].

(H3N8) $\left(\mathrm{K} 10,10^{7.7} \mathrm{EID}_{50} / \mathrm{ml}, \mathrm{Fc} 2\right)[7,8]$ were made and the detection limits of QCA and the two conventional RAD tests which were previously shown to be suitable for the diagnosis of EI, namely Quick Chaser Flu A, B (QC, Mizuho Medy, Saga, Japan) and BD Flu Examan (EMN, Becton, Dickinson and Co., Japan, Tokyo, Japan,) were determined as previously described [8]. Briefly, the detection limit $\left(\log (10) \mathrm{EID}_{50} / \mathrm{m} l\right)$ for each test was determined using $100 \mu l$ of a five-fold serial dilution of the virus stock according to the manufacturers' instructions. The results of QCA were judged by its exclusive reader (densitometer) [2]. The detection limits of QCA for M15 and $\mathrm{K} 10\left(10^{3.5} \mathrm{EID}_{50} / \mathrm{m} l\right.$ and $10^{4.2} \mathrm{EID}_{50} / \mathrm{m} l$, respectively) were five-fold lower than those of QC and EMN (Table 1), which indicates that QCA is able to detect lower titres of EIV than the conventional RAD tests.

To confirm this in clinical samples, four naïve one-year-old Thoroughbred fillies were inoculated with $\mathrm{M}_{15}\left(10^{7.9} \mathrm{EID}_{50} /\right.$ horse$)$ using an ultrasonic nebulizer on Day 0 as previously described [8]. Nasopharyngeal swabs were collected daily until Day 10 . The experimental protocols were approved by the Animal Care Committee of Equine Research Institute of Japan Racing Association. These nasopharyngeal swabs in addition to swabs collected from three naïve Thoroughbred horses experimentally infected with K10 in our previous study were tested by QCA [8]. The QCA results from the four horses infected with M15 and the three horses infected with K10 were summarized with the results of virus isolation (VI), and the results of 3 molecular diagnostic tests (conventional RT-PCR, real time (r) RT-PCR and RT-LAMP) [8], and two conventional RAD tests (QC and EMN) [8] in Tables 
2 and 3, respectively. The data obtained from the seven horses experimentally infected with M15 or K10 were pooled and the average detection periods of the tests were compared with repeated measures one-way ANOVA and Fisher's LSD test. The average detection period (days \pm standard deviation) for QCA (6.6 \pm 0.8$)$ was significantly longer than those of VI $(5.6 \pm 0.5, P=0.018)$ and the conventional RAD tests, QC $(5.4 \pm 0.8, P=0.015)$ and $\mathrm{EMN}(5.0 \pm 0.8, P=0.005)$. In contrast, no significant difference was observed between QCA and the three molecular tests (conventional RT-PCR $8.1 \pm 1.8, P=0.136$; rRT-PCR 8.3 $\pm 1.7, P=0.070$; RT-LAMP $8.1 \pm 1.8, P=0.091$ ). These findings indicated that the diagnostic sensitivity of QCA for EIV is higher than those of the conventional RAD tests. All the samples which were VI positive were judged to be positive by QCA. On Day 1 which was prior to the pyrexia onset, less than $50 \%$ of the 7 horses ( 1 or 3 horses) were diagnosed as positive by the conventional RAD tests despite the fact that they were shedding viable EIV as confirmed by VI. Such false-negative results could lead to an increased risk of missing horses during the incubation period if conventional RAD tests were used for screening during quarantine. The results indicate that QCA would be more beneficial than the conventional RAD test on an individual horse basis. In addition, 550 nasal swabs collected in 2015/16 from pyretic horses which had previously tested negative by the conventional RT-PCR also tested negative by QCA, indicating that the specificity of QCA is very high (100, 95\% confidence interval: 99.3-100\%). The results of QCA are automatically determined by a densitometry analysis machine [2], in contrast to the conventional RAD tests where the result is determined by eye and can be subjective. However, the QCA reader is able to process only one sample at a time (maximum $15 \mathrm{~min}$ ), and thereby limiting the utility of this test method for large scale surveillance during an EI outbreak. QCA is less sensitive than RT-PCR but could be used as a convenient screening test in a veterinary clinic or quarantine station.

In conclusion, our findings suggest that QCA is a highly specific method for diagnosing EI with a higher sensitivity than the conventional RAD tests.

\section{REFERENCES}

1. Cullinane, A. and Newton, J. R. 2013. Equine influenza-a global perspective. Vet. Microbiol. 167: 205-214. [Medline] [CrossRef]

2. Mitamura, K., Shimizu, H., Yamazaki, M., Ichikawa, M., Nagai, K., Katada, J., Wada, A., Kawakami, C. and Sugaya, N. 2013. Clinical evaluation of highly sensitive silver amplification immunochromatography systems for rapid diagnosis of influenza. J. Virol. Methods 194: 123-128. [Medline] [CrossRef]

3. Wada, A., Sakoda, Y., Oyamada, T. and Kida, H. 2011. Development of a highly sensitive immunochromatographic detection kit for H5 influenza virus hemagglutinin using silver amplification. J. Virol. Methods 178: 82-86. [Medline] [CrossRef]

4. Webster, R. G., Bean, W. J., Gorman, O. T., Chambers, T. M. and Kawaoka, Y. 1992. Evolution and ecology of influenza A viruses. Microbiol. Rev. 56: 152-179. [Medline]

5. Wilson, W. D. 1993. Equine influenza. Vet. Clin. North Am. Equine Pract. 9: 257-282. [Medline]

6. Yamanaka, T., Niwa, H., Tsujimura, K., Kondo, T. and Matsumura, T. 2008. Epidemic of equine influenza among vaccinated racehorses in Japan in 2007. J. Vet. Med. Sci. 70: 623-625. [Medline] [CrossRef]

7. Yamanaka, T., Cullinane, A., Gildea, S., Bannai, H., Nemoto, M., Tsujimura, K., Kondo, T. and Matsumura, T. 2015. The potential impact of a single amino-acid substitution on the efficacy of equine influenza vaccines. Equine Vet. J. 47: 456-462. [Medline] [CrossRef]

8. Yamanaka, T., Nemoto, M., Bannai, H., Tsujimura, K., Kondo, T., Matsumura, T., Gildea, S. and Cullinane, A. 2016. Evaluation of twenty-two rapid antigen detection tests in the diagnosis of Equine Influenza caused by viruses of H3N8 subtype. Influenza Other Respi. Viruses 10: $127-133$. [Medline] [CrossRef] 